\title{
Chronicles of a cancer foretold: 35 years of gastric cancer risk assessment
}

\author{
Massimo Rugge, ${ }^{1,2}$ Robert M Genta, ${ }^{3,4}$ David Y Graham, ${ }^{5}$ \\ Francesco Di Mario, ${ }^{6}$ Luiz Gonzaga Vaz Coelho, ${ }^{7}$ Nayoung Kim, \\ Peter Malfertheiner, ${ }^{9}$ Kentaro Sugano, ${ }^{10}$ Vladislav Tsukanov, ${ }^{11}$ \\ Pelayo Correa ${ }^{12}$
}

\section{INTRODUCTION}

In 1980, an international group of pathologists and clinicians led by Basil Morson published a critical review entitled Precancerous Conditions and Epithelial Dysplasia in the Stomach. ${ }^{1}$ This seminal article attempted to merge Eastern and Western pathological experiences in the evaluation of preneoplastic conditions into a systematic approach to the evaluation of gastric precancerous conditions. Thirty-five years after its publication, we pay homage to Professor Morson's team by revisiting their positions in light of the dramatic advances of our understanding of gastric disease that have occurred during this time. To this end, we have selected from the original article those statements that warranted reappraisal and, after reproducing them verbatim, have provided our comments.

\section{Introduction}

When considering problems of premalignancy, it is essential to define exactly what is meant by this term.

\footnotetext{
${ }^{1}$ Department of Medicine DIMED Pathology and Cytopathology Unit, University of Padova, Padova, Italy; ${ }^{2}$ Tumor Registry of the Veneto Region, Padova, Italy; ${ }^{3}$ Miraca Life Sciences Research Institute, Irving, Texas, USA; ${ }^{4}$ University of Texas Southwestern Medical School, Dallas, Texas, USA; ${ }^{5}$ Department of Medicine, Michael E. De Bakey Veterans Affairs Medical Center, and Baylor College of Medicine, Houston, Texas, USA; ${ }^{6}$ Department of Clinical and Experimental Medicine, University of Parma, Parma, Italy; ${ }^{7}$ Institute Alfa of Gastroenterology, Federal University of Minas Gerais, Belo Horizonte, Minas Gerais, Brazil; ${ }^{8}$ Department of Internal Medicine, Seoul National University Bundang Hospital, Seoungnam, Gyeonggi-do, South Korea; ${ }^{\circ}$ Department of Gastroenterology, Hepatology \& Infectious Diseases, Otto-von-Guericke University, University Medical Center of Magdeburg, Magdeburg, Germany; ${ }^{10}$ Department of Medicine, Jichi Medical University, Tochigi, Japan;

${ }^{11}$ State Scientific Medical Research Institute for Northern Problems of Siberian Division of Russian Academy of Medical Sciences, Krasnoyarsk, Russia; ${ }^{12}$ Department of Pathology, Microbiology and Immunology, Vanderbilt University Medical Center, Nashville, Tennessee, USA

Correspondence to Dr Massimo Rugge MD FACG, Surgical Pathology \& Cytopathology Unit, Department of Medicine DIMED, University of Padova, Via A. Gabelli, 61, Padova 35121, Italy; massimo.rugge@unipd.it
}

\begin{abstract}
A distinction can be made between a precancerous condition and a precancerous lesion. The former is best regarded as a clinical state associated with a significantly increased risk of cancer, whereas a precancerous lesion is a histopathological abnormality in which cancer is more likely to occur than in its apparently normal counterpart. In many clinical conditions with an increased risk of cancer, there is also an identifiable precancerous lesion, but this is not invariably so.
\end{abstract}

Studies carried out in the last three decades have supported the multifactorial aetiology of sporadic gastric cancer and have led to the concept of a progressive 'cascade' of phenotypic and molecular changes that may eventually result in neoplasia. $^{2}$ In 1994, Helicobacter pylori was added to the cascade, providing the hitherto missing crucial aetiological element that initiates the entire process. ${ }^{3}{ }^{4}$ While the distinction between precancerous condition and lesion remains useful, the current understanding of gastric oncogenesis (including its early molecular disarrangements) begs an expansion of the concept of 'lesion', which should not only refer to focal changes (eg, dysplasia) as envisioned by Morson, but should also cover the spectrum of the organic (histological and molecular) abnormalities associated with an increased gastric cancer risk. In keeping with the concept that both $H$. pylori gastritis and autoimmune gastritis confer a small, but measurable increased risk for gastric cancer, we suggest that they be considered part of the spectrum of early precancerous lesions (the former Morson's conditions). ${ }^{5}$ Intraepithelial neoplasia (Morson's dysplasia) can be viewed as the most advanced stage of the premalignant cascade (table 1; figure 1).

Lesions arising from mucosal-associated lymphoid tissue (MALT) should now be viewed as part of a continuum that may progress to a lymphoid malignancy. At the time of Morson's writing, MALT lymphomas (more recently renamed as marginal zone B cell lymphomas) were categorised as pseudo-lymphomas, a heterogeneous spectrum of histological abnormalities, including lymphoid hyperplasia, lymphoid proliferation 'at the interface between benign and malignant' and low-grade lymphoid neoplasia. Current evidence is unequivocal about the preneoplastic nature of these lesions. ${ }^{6-8}$

With the exception of autoimmune atrophic gastritis, whose aetiology remains uncertain (see below), the common denominator in the prevention of all precancerous lesions as defined above is the eradication of $H$. pylori.

\section{Atrophic gastritis}

This is a histological diagnosis. The main features are a variable degree of inflammation, atrophy of gastric glands, and often associated intestinal metaplasia, with changes more commonly found in the antrum than in the body or the fundus of the stomach....

Most intestinal-type gastric cancers develop in atrophic mucosa; thus, the assessment of gastric mucosal atrophy is a crucial step in the identification of at-risk patients. ${ }^{9}{ }^{10}$ Although we are in agreement with Morson's statement that atrophic gastritis 'is a histological diagnosis', the last 35 years have witnessed the development of new technologies that, while not replacing histology, provide useful adjuncts to the detection of gastric atrophy. The most notable are new endoscopic instruments that provide a view of the gastric mucosa unimaginable 35 years ago. Magnification endoscopy, autofluorescence, narrow-band imaging and blue-laser imaging allow endoscopists to gather reliable information on the distribution and extension of atrophy, thus helping to better select areas for biopsy sampling. ${ }^{11-13}$

The loss of gastric glands can be functionally tested by measuring their circulating products. Serum pepsinogen I (PgI) and pepsinogen II (PgII) are the most reliable markers of functional mucosal atrophy: PgI, synthesised and secreted by chief cells, reflects the functional status of the oxyntic mucosa; PgII is produced by both oxyntic and antral mucosa. Multiple studies have shown a lower PgI/PgII ratio in patients with corpus atrophy, irrespective of its aetiology. ${ }^{14} 15$ When integrated with the serological evaluation of antibodies against Helicobacter, parietal cells and intrinsic factor, the Pg values and their ratio can provide a non-invasive and affordable estimate of gastric function and morphology. Some authors have referred to this battery of tests as the 'serological biopsy'. While the serological biopsy now fills a useful 
Table 1 Gastric precancerous Conditions and Lesions

\begin{tabular}{ll}
\hline Precancerous conditions & Precancerous lesions \\
\hline H. pylori-associated gastritis & Early precancerous lesions \\
Autoimmune gastritis & (H. pylori-associated gastritis, Autoimmune gastritis) \\
Gastric (chronic) ulcer & Gastritis Stage O \\
Gastric stumps & Gastritis Stage I \\
Syndromic neoplastic diseases: & Gastritis Stage II \\
$\quad$ Li-Fraumeni syndrome & Gastritis Stage III \\
Hereditary breast and ovarian cancer & Gastritis Stage IV \\
Lynch Syndrome & Advanced precancerous lesions \\
$-\quad$ Juvenile polyposis syndrome & (H. pylori, Autoimmune, Syndromic neoplasia) \\
PTEN hamartoma tumour syndrome (PHTS) & $>$ Intra-Epithelial Neoplasia Low-grade (IEN-LG) \\
& $>$ Intra-Epithelial Neoplasia High-grade (IEN-HG) \\
\hline
\end{tabular}

Precancerous Conditions: clinically-defined diseases associated to increased (gastric and non-gastric) cancer risk, in which precancerous lesions may occur.

Precancerous Lesions: organic modification of the native mucosal status (at both phenotypic and molecular levels). Gastritis stages recapitulate the multistep inflammatory/atrophic involvement of the gastric mucosa (mainly due to Helicobacter infection): their precancerous meaning is expected to increase along with the severity of the mucosa atrophy, as expressed by the gastritis stage.

Advanced precancerous lesions are basically focal, and mainly restricted to patients harboring extensive atrophic lesions (high-risk stages; Stages III and IV).

MUTYH-gene (located on chromosome 1p35); PTEN-gene (located on chromosome 10q22-23).

niche in epidemiological studies, both endoscopic and histological assessments of atrophy continue to play the central role in clinical practice. Since the staging of atrophy and the determination of its aetiology are necessary steps in the evaluation of cancer risk, biopsy sets representative of both antrum and corpus are indispensable. The sampling protocols proposed by the Updated Sydney System and subsequently endorsed by both the Operative Link for Gastritis Assessment (OLGA) and the Operative Link on Gastric Intestinal Metaplasia (OLGIM) staging systems are adequate and are most useful when accompanied by sampling of any visible lesions. ${ }^{16-19}$

Patients with atrophic gastritis are statistically at increased risk from cancer of the stomach, but precise measurement of this risk is yet to be determined.
The OLGA Staging System is an instrument built on the natural history of gastric atrophy and its associated cancer risk. Proposed in 2005 by an international group of gastroenterologists and pathologists, ${ }^{17-19}$ it borrows its staging framework from the oncology vocabulary ${ }^{20}$ and ranks the histological phenotypes of gastritis along a scale (0-IV) of increasing mucosal atrophy. The cancer risk associated with stages 0 , I and II, while not absent, ${ }^{5}$ is extremely low, while stages III and IV (characterised by extensive atrophy of both antral and oxyntic mucosa) are associated with high risk of intestinal-type gastric cancer. An alternative staging method proposed as a modification of the OLGA System (OLGIM) bases its stage assessment exclusively on the extension of IM, as assessed in both antral and oxyntic biopsy samples. ${ }^{19}$ While both systems have strengths and weaknesses, they have proved useful in the prognostication of cancer risk by allowing the placement of patients at approximate points along the path where chronic gastritis advances from the reversible inflammatory lesions (generally most severe in the antrum) to the extensive atrophic changes involving both antrum and corpus. ${ }^{21} 22$ While we still lack large-scale prospective population studies, the available information consistently associates a negligible risk of cancer with the 0-I-II stages. Because high-risk stages (III-IV) correlate with low serum levels of Pgs (particularly PgI), and low PgI/PgII ratios, Pg serology has been proposed as a non-invasive method for the initial assessment of atrophy in clinical practice. ${ }^{18} 23$ The clinical reliability of the morphological versus functional correlation still deserves to be further addressed.

Within the histological spectrum of atrophic gastritis intestinal metaplasia is the most sensitive risk indicator...

This statement, which represents the rationale for the OLGIM System, remains fundamentally valid: the extent of metaplastic transformation, particularly when combined with histochemical subtyping, parallels the cancer risk. ${ }^{24}$ The metaplastic transformation of the native gastric glands results into a loss of appropriate glands; hence, the inclusion of metaplasia in the definition of gastric mucosa atrophy. $^{26}$ Native gastric glands can be replaced by two types of structures: (i) intestinal-type glands (enterocytes, goblet and Paneth cells; ie, IM) and (ii) pylorictype glands ('pseudo-pyloric metaplasia'). IM has traditionally been classified into small intestinal type, with well-spaced goblet cells and a brush border lining the enterocytes, and colonic type, with a more disorderly architecture and no brush border. $^{27}$

Figure 1 Model of Helicobacter pylori-associated lesions according to gastric oncogenetic cascade. The organic lesions are also expressed in terms of gastritis stage. Grey, normal mucosa; Green, H. pylori; Light red, mucosa inflammation; Yellow, mucosa atrophy (with or without intestinal metaplasia (IM)); Violet, advanced precancerous lesion (intraepithelial neoplasia) at the angularis incisura. 
By staining mucins with high iron diamine, IM has been further subtyped as Type I (complete or small intestinal type) and Types II and III (incomplete or colonic type). Several studies have associated colonic type IM (mostly III) with the highest risk for neoplastic transformation. However, because the proportion of Type III metaplasia is exponentially related to the extent of mucosal intestinalisation, ${ }^{25}$ the histology score of IM (as assessed by appropriate endoscopic biopsy mapping) can be considered as related to the cancer risk. Recently, it has been demonstrated that the high-risk gastritis stages (OLGA stages III and IV) feature mostly Type II and III IM, which incorporates the prognostic message obtainable from histochemical gastric mucin subtyping within the staging messages. ${ }^{21}$

Pseudo-pyloric metaplasia, now referred to as spasmolytic polypeptide-expressing metaplasia (SPEM), is the metaplastic replacement of oxyntic glands by mucinsecreting antral-like glands. ${ }^{28}{ }^{29}$ The identification of SPEM, which occurs only in the oxyntic mucosa, is contingent upon the accurate topographic identification of the sample. SPEM, found in virtually all forms of atrophic gastritis, also represents the earliest phase of the atrophic transformation in autoimmune gastritis. In H. pylori infection, the cephalad spreading of the mucosal damage is associated with an enlarging 'lawn' of metaplastic pyloric-type epithelium, which commonly coexists with the intestinalisation of the distal gastric mucosa. Studies in animal models have confirmed SPEM as the first expression of atrophy and have helped elaborate hypotheses regarding its role in the development of $\mathrm{IM}^{28}$

\section{Pernicious anaemia \\ There is statistical and histological evi- dence that patients with pernicious anaemia are at increased risk from cancer of the stomach. True adenomatous polyps and carcinoma of the stomach have been reported to be three to four times more common in patients with pernicious anaemia than in the general population, although a recent study suggests that this may be an underestimate, since some patients with carcinoma but without overt pernicious anaemia are in a "pre- pernicious anaemia stage.}

Pernicious anaemia is the result of long-standing cobalamin deficiency caused by advanced oxyntic mucosal atrophy. ${ }^{30} 31$ In its original description, this corpus-restricted atrophic gastritis was assumed to be primarily due to an autoimmune attack directed at parietal cells. In the last decades, however, atrophy has been most consistenly associated with long standing H.pylori infection. In addition, it has been suggested that $H$. pylori infection might also trigger and sustain an immune-mediated attack against the proton pump, resulting in organic lesions (ie, corpus-restricted atrophic gastritis) identical to those of primary gastric autoimmunity. ${ }^{32}$

This widened pathogenetic spectrum of oxyntic atrophy should also include the overlap of primary and secondary (postinfectious) autoimmune conditions. Because it is difficult to reliably discriminate between primary and secondary autoimmune corpus atrophy in epidemiological studies, both impact and magnitude of the cancer risk associated with each of its variants remain unknown. ${ }^{33} \mathrm{~A}$ study based on the OLGA staging suggests that the risk for precancerous lesions and cancer is only increased when autoimmune gastritis coexists, or has coexisted, with H. pylori infection. ${ }^{34}$ However, researchers from the US National Cancer Institute have recently provided evidence that subjects with pernicious anaemia are at increased risk for non-cardia gastric cancers and carcinoids. ${ }^{35}$ While this elegant study supports recent recommendations to consider surveillance in subjects with advanced atrophy and metaplasia, irrespective of pernicious anaemia, ${ }^{36}$ it does not address the question of whether the gastric cancer risk in patients with atrophic gastritis is related to previous exposure to Helicobacter.

Recent data suggest that SPEM associated with the early phase of corpusrestricted autoimmune gastritis may progress to more advanced precancerous lesions, but the SPEM-associated cancer risk remains unknown. ${ }^{37}$

\section{Gastric polyps \\ Polypoid lesions of the stomach can be divided into those with and without malignant potential. The common ones, hyperplastic or regenerative polyps, have insignificant malignant potential. Polypoid lesions in which the epithelium shows dysplasia (which are called aden- omas or borderline lesions by some) have a significant capacity for malignant change.}

The proportions of the different types of gastric polyps encountered at esophagogastric-duodenoscopy (EGD) have changed substantially in the last three decades. ${ }^{38} 39$ The industrialised world has experienced a progressive decrease of $H$. pylori infection and its related conditions, the use of proton pump inhibitors (PPI) has become widespread and indications for esophago-gastricduodenoscopy have placed a greater emphasis on GORD, Barrett's oesophagus and the early detection of oesophageal adenocarcinoma. Thus, while Morson's major concerns lay in the detection of adenomas (dysplastic lesions with malignant potential) and hyperplastic polyps (even then recognised as having insignificant malignant potential'), today more than $80 \%$ of the polyps detected in Western countries consist of fundic gland polyps in the vast majority of cases associated with chronic proton pump inhibitor use. In countries with high $H$. pylori prevalence, most polyps are of the hyperplastic-inflammatory type. $^{38} 39$

Whereas non-neoplastic epithelial polyps have only a minimal influence on gastric cancer risk, sporadic neoplastic (adenomatous) polyps have a biological and clinical behaviour similar to that of neoplastic intraglandular lesions arising in flat (non-rising, non-polypoid) mucosa. The larger an adenomatous polyp, the greater its risk of containing foci of adenocarcinoma. Synchronous adenocarcinomas in other areas of the stomach have been reported in up to $30 \%(23 / 77)$ of patients with adenomas containing foci of invasive cancer. ${ }^{40}$ Thus, although the relative prevalence of the different types of polyps has changed, Morson's views regarding the cancer risk of gastric (adenomatous) polyps remain valid today.

\section{Epithelial dysplasia \\ Attention has so far been concentrated on the above precancerous conditions and much less emphasis has been placed on epithelial dysplasia in the stomach as a marker for increased cancer risk. It is important that dysplasia should be defined and its significance evaluated as a possible marker common to all the above precancerous conditions. The main histological and cytological fea- tures of epithelial dysplasia are cellular atypia, abnormal differentiation, and disorganised mucosal architecture. These can occur in ordinary gastric (foveolar) epithelium as well as in intestinal meta- plasia, both of which may be the source of carcinoma.}

Morson and colleagues described gastric dysplasia as a precancerous lesion resulting from the concurrence of three main cytological and histological changes: (i) epithelial atypia; (ii) loss of epithelial commitment and (iii) disrupted mucosal architecture. In the Japanese literature, similar lesions had been labelled as borderline lesions, atypical epithelium or group 3 lesions. ${ }^{41}$

Since the early 1990s, a growing body of molecular studies have detected genotypic changes in dysplastic epithelia that 
are also found in invasive carcinomas. Based on both their cancer-like morphological abnormalities and their mutated molecular profiles, dysplastic epithelia were reclassified as neoplastic. This resulted in the creation of two major categories within the spectrum of neoplastic disease: (i) non-invasive neoplasia (formerly, dysplasia) and (ii) the invasive/infiltrative neoplasia. In the gastric oncogenic cascade, non-invasive neoplasia (synonyms: intraepithelial, intraglandular) represents the biological bridge between early precancerous lesions (atrophy and metaplasia) and early gastric cancer. ${ }^{42} 43$ Subsequently, intraepithelial neoplastic lesions were further segmented in two clinicobiological grades (low-grade and high-grade), the latter with a greater risk of progression to invasive cancer.

Different classifications of neoplastic intraepithelial lesions have been proposed. Both the Padova International ${ }^{44}$ and the WHO classification ${ }^{45}$ specifically addressed the precancerous lesions arising in gastric mucosa. The Vienna classification has proven to be problematic in its attempt to provide a pan-GI tract system to classify all precancerous diseases occurring in the anatomically diverse structures between the oral cavity and the anal mucosa. ${ }^{46}$

\section{Clinical implications and further research \\ It is important to realise that the degree of risk associated with epithelial dysplasia is not yet established. Severe dysplasia on its own is not an indication for surgical intervention. However, experience of epithelial dysplasia in other organs suggests the importance of close follow-up for individual patients (including endoscopy and biopsy).}

Significant progress has been made in our understanding of the course of advanced precancerous lesions owing to the invaluable contribution of a number of long-term follow-up studies. Solid evidence supports the promoting role of $H$. pylori, even in the advanced stages of the precancerous cascade. Accordingly, eradication is recommended even in the presence of advanced lesions. ${ }^{47}$ Low-grade intraepithelial lesions are known to be associated with a risk of cancer progression that increases in the presence of both $H$. pylori infection and extensive atrophy or metaplasia (highstage gastritis). This evidence provides the rationale for endoscopic follow-up. The cancer risk associated with a validated diagnosis of a high-grade lesion (ie, confirmed by another experienced histopathologist) is extremely high and represents an indication for resection, now made much less invasive by the availability of endoscopic mucosal and submucosal resection.

More than 15 years ago, linkage analysis implicated germline mutations in the CDH1 tumour-suppressor gene (encoding the protein E-cadherin) as primary involved in the onset of hereditary signet-ring gastric carcinoma. The extremely high prevalence of both stomach and breast cancers in these patients currently suggests gastrectomy as the only secondary prevention strategy: molecular disarrangements, however, are probably more extensively involved in gastric cancer pathogenesis, even in its sporadic variants. How both host and environmental components may interact in modulating cancer risk will likely dominate the gastric cancer research landscape for the next 35 years. $^{48}$

\section{CONCLUSIONS}

The biological, diagnostic and therapeutic panorama has dramatically changed in the 35 years since the publication of Morson's seminal paper. The discovery of $H$. pylori has revolutionised practice of gastroenterology and the script of Correa's oncogenic cascade has found its lead actor (figure 1). Lesions and conditions viewed as idiopathic in Morson's times are now known to be caused by $H$. pylori infection and have become curable. The recent interest in large, even nationwide $H$. pylori eradication programmes as a mean to eliminate gastric cancer will require implementation of this strategy at the population level and will have to be fully integrated into national healthcare priorities. The revised distinction between early and advanced precancerous lesions and the recognition that the extension of metaplastic atrophy is a reliable marker for gastric cancer risk have made possible more targeted and cost-effective surveillance strategies. These should be implemented using efficient delivery systems with a timely referral for positive test. Such measures, coupled with the wide availability of standardised treatment regimens based on clinical efficacy, side effects, simplicity, duration and cost, could make the near eradication of gastric cancer an achievable goal in many parts of the world. ${ }^{49} 50$

Contributors All authors participated in writing the manuscript and approved the final, submitted version.

Funding This work was partly supported by a grant from the Italian Association for Cancer Research (AIRC Regional grant 2008 N. 6421). This study was conducted under the cooperative auspices of the Healthy Stomach Initiative (HSI).
Competing interests None declared.

Ethics approval Padova University, Italy

Provenance and peer review Not commissioned; externally peer reviewed.
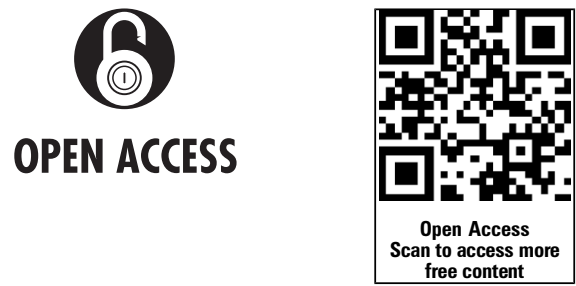

Open Access This is an Open Access article distributed in accordance with the Creative Commons Attribution Non Commercial (CC BY-NC 4.0) license, which permits others to distribute, remix, adapt, build upon this work non-commercially, and license their derivative works on different terms, provided the original work is properly cited and the use is noncommercial. See: http://creativecommons.org/licenses/ by-nc/4.0/

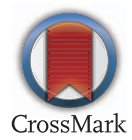

To cite Rugge M, Genta RM, Graham DY, et al. Gut 2016;65:721-725.

Received 4 October 2015

Revised 9 December 2015

Accepted 15 December 2015

Published Online First 29 February 2016

Gut 2016;65:721-725.

doi:10.1136/gutjnl-2015-310846

\section{REFERENCES}

1 Morson BC, Sobin LH, Grundmann E, et al. Precancerous conditions and epithelial dysplasia in the stomach. J Clin Pathol 1980;33:711-21.

2 Correa P. A human model of gastric carcinogenesis Cancer Res 1988;48:3554-60.

3 [No authors listed]. Infection with Helicobacter pylori. IARC Monogr Eval Carcinog Risks Hum 1994;61:177-240.

4 Hunt RH, Camilleri M, Crowe SE, et al. The stomach in health and disease. Gut 2015;64:1650-68.

5 Uemura N, Okamoto S, Yamamoto S, et al. Helicobacter pylori infection and the development of gastric cancer. N Engl J Med 2001;345:784-9.

6 Ruskoné-Fourmestraux A, Fischbach W, Aleman BM, et al. EGILS consensus report. Gastric extranodal marginal zone B-cell lymphoma of MALT. Gut 2011;60:747-58.

7 Sena Teixeira Mendes L, D Attygalle A, C Wotherspoon A. Helicobacter pylori infection in gastric extranodal marginal zone lymphoma of mucosa-associated lymphoid tissue (MALT) lymphoma: a re-evaluation. Gut 2014;63:1526-7.

8 Nakamura S, Sugiyama T, Matsumoto T, et al. Long-term clinical outcome of gastric MALT lymphoma after eradication of Helicobacter pylori: a multicentre cohort follow-up study of 420 patients in Japan. Gut 2012:61:507-13.

9 Ferlay J, Soerjomataram I, Dikshit R, et al. Cancer incidence and mortality worldwide: sources, methods and major patterns in GLOBOCAN 2012. Int J Cancer 2015;136:E359-86.

10 Park YH, Kim N. Review of atrophic gastritis and intestinal metaplasia as a premalignant lesion of gastric cancer. J Cancer Prev 2015;20:25-40. 
11 Saka A, Yagi K, Nimura S. OLGA- and OLGIM-based staging of gastritis using narrow-band imaging magnifying endoscopy. Dig Endosc 2015;27:734-41.

12 Kaise M, Kato M, Tajiri H. High-definition endoscopy and magnifying endoscopy combined with narrow band imaging in gastric cancer. Gastroenterol Clin North Am 2010;39:771-84.

13 Okubo M, Tahara T, Shibata T, et al. Usefulness of magnifying narrow-band imaging endoscopy in the Helicobacter pylori-related chronic gastritis. Digestion 2011;83:161-6.

14 Lee JY, Kim N, Lee HS, et al. Correlations among endoscopic, histologic and serologic diagnoses for the assessment of atrophic gastritis. J Cancer Prev 2014;19:47-55.

15 Lomba-Viana R, Dinis-Ribeiro M, Fonseca $F$, et al. Serum pepsinogen test for early detection of gastric cancer in a European country. Eur J Gastroenterol Hepatol 2012;24:37-41.

16 Dixon MF, Genta RM, Yardley JH, et al. Classification and grading of gastritis. The updated Sydney System. International Workshop on the Histopathology of Gastritis, Houston 1994. Am J Surg Pathol 1996;20:1161-81.

17 Rugge M, Genta RM. Staging gastritis: an international proposal. Gastroenterology 2005;129:1807-8.

18 Rugge M, de Boni M, Pennelli G, et al. Gastritis OLGA-staging \& gastric cancer risk: a twelve year clinico-pathological follow-up study. Aliment Pharmacol Ther 2010;31: 1104-11.

19 Capelle LG, de Vries AC, Haringsma J, et al. The staging of gastritis with the OLGA system by using intestinal metaplasia as an accurate alternative for atrophic gastritis. Gastrointest Endosc 2010;71:1150-8.

20 Sobin LH, Wittekind C. TNM classification of malignant tumours. 6th edn. New York: Wiley-Liss, 2002.

21 Rugge M, Fassan M, Pizzi M, et al. Operative link for gastritis assessment vs operative link on intestinal metaplasia assessment. World J Gastroenterol 2011;17:4596-601.

22 Isajevs S, Liepniece-Karele I, Janciauskas D, et al. Gastritis staging: interobserver agreement by applying OLGA and OLGIM systems. Virchows Arch 2014:464:403-7.

23 Tu H, Sun L, Dong $X$, et al. Temporal changes in serum biomarkers and risk for progression of gastric precancerous lesions: a longitudinal study. Int J Cancer 2015;136:

425-34.

24 Jass JR, Filipe MI. The mucin profiles of normal gastric mucosa, intestinal metaplasia and its variants and gastric carcinoma. Histochem J 1981;13: 931-9.

25 Cassaro M, Rugge M, Gutierrez 0, et al. Topographic patterns of intestinal metaplasia and gastric cancer. Am J Gastroenterol 2000;95:1431-8.

26 Rugge M, Correa P, Dixon MF, et al. Gastric mucosal atrophy: interobserver consistency using new criteria for classification and grading. Aliment Pharmacol Ther 2002;16:1249-59.

27 Jass JR, Walsh MD. Altered mucin expression in the gastrointestinal tract: a review. J Cell Mol Med 2001;5:327-51.

28 Goldenring JR, Nam KT, Wang TC, et al. Spasmolytic polypeptide-expressing metaplasia and intestinal metaplasia: time for reevaluation of metaplasias and the origins of gastric cancer. Gastroenterology 2010;138:2207-10.

29 Sousa JF, Nam KT, Petersen CP, et al. miR-30-HNF4gamma and miR-194-NR2F2 regulatory networks contribute to the upregulation of metaplasia markers in the stomach. Gut Published Online First: 23 Mar 2015. doi:10.1136/ gutjnl-2014-308759

30 Strickland RG, Mackay IR. A reappraisal of the nature and significance of chronic atrophic gastritis. Am J Dig Dis 1973;18:426-40.

31 Neumann WL, Coss E, Rugge $M$, et al. Autoimmune atrophic gastritis--pathogenesis, pathology and management. Nat Rev Gastroenterol Hepatol 2013;10:529-41.

32 Zhang Y, Weck MN, Schöttker B, et al. Gastric parietal cell antibodies, Helicobacter pylori infection, and chronic atrophic gastritis: evidence from a large population-based study in Germany. Cancer Epidemiol Biomarkers Prev 2013;22:821-6.

33 Genta RM, Rugge M. Atrophic gastritis in the arctic. Clin Gastroenterol Hepatol 2015;13:1601-3.

34 Rugge M, Fassan M, Pizzi M, et al. Autoimmune gastritis: histology phenotype and OLGA staging. Aliment Pharmacol Ther 2012;35:1460-6.

35 Murphy G, Dawsey SM, Engels EA, et al. Cancer risk after pernicious anemia in the US elderly population. Clin Gastroenterol Hepatol 2015:13:2282-9.e4.

36 Kuipers EJ. Pernicious anemia, atrophic gastritis, and the risk of cancer. Clin Gastroenterol Hepatol 2015;13:2290-2.
37 Weis VG, Sousa JF, LaFleur BJ, et al. Heterogeneity in mouse spasmolytic polypeptide-expressing metaplasia lineages identifies markers of metaplastic progression. Gut 2013;62:1270-9.

38 Carmack SW, Genta RM, Schuler CM, et al. The current spectrum of gastric polyps: a 1-year national study of over 120,000 patients. Am J Gastroenterol 2009;104:1524-32.

39 Shaib YH, Rugge M, Graham DY, et al. Management of gastric polyps: an endoscopy-based approach. Clin Gastroenterol Hepatol 2013;11:1374-84.

40 Park do Y, Lauwers GY. Gastric polyps: classification and management. Arch Pathol Lab Med 2008; 132:633-40.

41 Aiko T, Sasako M. The new Japanese classification of gastric carcinoma: points to be revised. Gastric Cancer 1998;1:25-30.

42 Rugge M, Farinati F, Di Mario F, et al. Gastric epithelial dysplasia: a prospective multicenter follow-up study from the Interdisciplinary Group on Gastric Epithelial Dysplasia. Hum Pathol 1991;22: 1002-8.

43 Rugge M, Cassaro M, Di MF, et al. The long term outcome of gastric non-invasive neoplasia. Gut 2003;52:1111-16.

44 Rugge M, Correa P, Dixon MF, et al. Gastric dysplasia: the Padova international classification. Am J Surg Pathol 2000;24:167-76.

45 Lauwers GY, Carneiro F, Graham DY, et al. WHO Classification of Tumors of the Digestive System. Tumours of the stomach: gastric carcinoma. In: Bosman FT, Carneiro F, Hruban RH, et al, eds. 4th edn. Lyon: IARC, 2010:48-58.

46 Schlemper RJ, Riddell RH, Kato Y, et al. The Vienna classification of gastrointestinal epithelial neoplasia. Gut 2000;47:251-5.

47 Rugge M, Capelle LG, Cappellesso R, et al. Precancerous lesions in the stomach: from biology to clinical patient management. Best Pract Res Clin Gastroenterol 2013;27:205-23.

48 van der Post RS, Vogelaar IP, Carneiro F, et al. Hereditary diffuse gastric cancer: updated clinical guidelines with an emphasis on germline CDH1 mutation carriers. J Med Genet 2015;52: 361-74.

49 Malfertheiner P, Megraud F, O'Morain CA, et al. Management of Helicobacter pylori infection-the Maastricht IV/Florence Consensus Report. Gut 2012;61:646-64.

50 Rugge M. Secondary prevention of gastric cancer. Gut 2007;56:1646-7. 